\title{
Análisis bibliométrico de la producción científica de un investigador educativo adscrito al Sistema Nacional de Investigadores
}

\author{
Mauro Rodríguez Jiménez ${ }^{1}$ \\ Juana May Landero ${ }^{2}$
}

Recepción: 10-10-2017 / Aceptación: 03-12-2018

\section{Resumen}

El artículo de investigación analiza la producción científica de un investigador educativo adscrito al Sistema Nacional de Investigadores de México, a través de un estudio descriptivo de los resultados obtenidos a partir de un análisis bibliométrico de la producción científica del investigador, desde el año 1991 a 2017. Para el análisis se realizaron búsquedas en distintas bases de datos para localizar artículos, capítulos de libros y libros donde el investigador se presenta como autor, coautor o coordinador.

La productividad del investigador se calculó de acuerdo a las tipologías documentales (revistas científicas/académicas y editoriales); la producción por año, por país de publicación y las temáticas más frecuentes. En total se trabajó con un corpus de 103 documentos, de los cuales 51 fueron artículos académicos/científicos, 23 capítulos de libros y 29 libros. Los resultados obtenidos revelaron los años de mayor productividad del profesor-investigador siendo los años 2008, 2010, 2011 y 2016, respectivamente, los que tuvieron mayor índice de publicaciones. Las revistas y editoriales usadas con mayor regularidad corresponden a Magistralis en artículos; GERNIKA y Trillas en los capítulos de libros y en libros. Las temáticas abordadas con mayor frecuencia se relacionan con la Educación, la Ética y los Valores.

Palabras clave: producción científica, profesor investigador, análisis bibliométrico, indicadores bibliométricos, educación.

\begin{abstract}
This research article analyses the scientific production of an educational research assigned to the National System of Researchers from Mexico by a descriptive study of the results obtained from a bibliometric analysis of the scientific production of the researcher, from the year 1991 until the year 2017. For the analysis, some research was made in different databases to find articles, book chapters and books in which the researcher is presented as the author, co-author or coordinator.

The researcher productivity was calculated based on documentary typologies (scientific magazines/ academic and editorials); production per year, per country of publication and the most frequent topics. In total, we worked with a corpus of 103 documents, in which 51 were academic/ scientific articles, 23 book chapters and 29 books. The results obtained revealed the years of greatest teacher-researcher productivity were the years 2008, 2010, 2011 and 2016, respectively, the ones that obtained the highest publication ratings. The magazines and editorials used regularly belong to Magistralis in articles; GERNIKA and Trillas in book chapters and in books. The most frequently addressed topics are related to Education, Ethics and Values.
\end{abstract}

Key words: Scientific production, professor researcher, bibliometric analysis, bibliometric indicators, education.

1. Licenciatura en Idiomas, Universidad Juárez Autónoma de Tabasco, México; email: 122J16031@egresados.ujat.mx

2. Maestra en Educación, Profesora-Investigadora de la DAEA, Universidad Juárez Autónoma de Tabasco, México; email: educationphd2017@gmail.com 


\section{Introducción}

Los investigadores tienen un papel importante en el desarrollo del conocimiento en las distintitas áreas de la ciencia. Actualmente publican sus estudios y nuevos descubrimientos en revistas especializadas con el fin de difundirlos a la comunidad científica. Estas contribuciones a las distintitas áreas del saber tienen un crecimiento tal que pueden ser objeto de análisis y dan pautas para realizar estudios bibliométricos $(\mathrm{EB})^{3}$ para evaluar la actividad científica de revistas e instituciones; y también, en micro, hacer análisis de los indicadores que describen la trayectoria y producción científica de quienes la realizan.

La bibliometría "constituye un eslabón fundamental dentro del proceso de investigación y por tanto, se ha convertido en una herramienta que permite calificar la calidad del proceso generador de conocimiento y el impacto de este proceso en el entorno" (Rueda-Clausen, Villa-Roel, \& Rueda-Clausen, 2005, págs. 29-36).

Los estudios bibliométricos se realizan generalmente en revistas, países e instituciones considerando tener un impacto mayor; por ejemplo, en el estudio "Análisis bibliométrico de los artículos originales publicados en la revista Universitas Scientiarum" por Tatiana Alexandra Escorcia-Otálora y Raúl Alberto Poutou-Piñales se analiza la productividad científica de una revista a partir de la productividad, colaboración con otras revistas, conexiones entre autores, etc. En estudios realizados en instituciones se puede mencionar el "Análisis bibliométrico de la producción científica de la Universidad Politécnica de Valencia 1973-2001" de Adolfo Alonso Arroyo, Antonio Pulgarín, Isidoro Gil-Leiva; estudio donde se realiza el análisis a partir de artículos y congresos tomando en cuenta la productividad, distribución por tiempo, por tipo de documento e idioma.

La bibliometría se define como "una disciplina con alcance multidisciplinario que utiliza distintos indicadores cuantitativos y cualitativos para analizar la producción científica de un grupo de autores, mide el crecimiento de determinadas áreas, estudia las tendencias de publicación, etc." (Díaz Mujica, 2007, pág. 22). Los EB se realizan en las ciencias sociales aplicando métodos estadísticos y matemáticos que permiten definir los procesos de la comunicación escrita, y de la misma forma medir el desarrollo de las distintitas disciplinas científicas mediante técnicas de recuento, resultando un método confiable y universal para medir la productividad.

Los estudios bibliométricos son de gran utilidad pues

tienen un gran valor dentro de la literatura científica, por cuanto orientan al lector en torno a diversos asuntos académicos, tales como las temáticas que mayor acogida tienen en determinada revista, o el índice de colaboración que hay en los artículos que se publican, etc. (Agudelo, Bretón López, \& Buela Casal, 2003, pág. 507).

3. Los autores utilizarán estas siglas dentro del artículo para referirse al término estudios bibliométicos. 
Estudios recientes como el "Análisis bibliométrico sobre la producción científica archivística en la Red de Revistas Científicas de América Latina" (Montilla Peña, 2012, pág. 1) y "Bibliometría y ciencias sociales" (Rubio, 2012) dividen la bibliometría en dos áreas:

a. Descriptiva, que se ocupa de aspectos cuantitativos, tales como la distribución temática, documental, geográfica, y su productividad.

b. Evaluativa, que agrega a la bibliometría descriptiva otros estudios de evaluación de la actividad científica.

Un análisis bibliométrico se basa en parámetros e indicadores mediante los cuales se evalúa la actividad científica, tomando como objeto de estudio las investigaciones de cualquier disciplina transmitidas en forma de publicaciones. Algunos parámetros pueden ser la productividad autoral que mide el número de publicaciones producidas por un investigador, un grupo de investigadores, una institución o un país, "Los estudios de productividad por autores dan como resultado la existencia de un pequeño grupo de personas muy productivas al lado de un gran número que apenas publican” (Lotka, 1926, pág. 317). En ciertos casos puede que un autor trabaje en co-autoría; y en tal caso, también es posible hacer un análisis del grado de colaboración de los autores, lo que correspondería a otro parámetro llamado productividad de colaboración autoral.
Otro parámetro sujeto a análisis es la productividad por instituciones y editoriales, mismos que van de la mano con los lugares de edición. "El estudio de las instituciones públicas o privadas responsables y su tipología permite obtener un panorama sobre los "circuitos" de edición en los que se mueve una disciplina” (Documentación, 1986-1987). También puede ser en función de su temática la cual puede tener aspectos interesantes, aunque resulta más complejo. La información de este parámetro se obtiene a través de las palabras clave y/o resúmenes que aparecen en las publicaciones.

La productividad por año resulta de utilidad para medir el aumento o disminución de la producción científica. Para su análisis es necesario tener en cuenta diversos factores que pueden influir en el resultado "El aumento de publicaciones es claro en cualquiera de las Ciencias Sociales, pero a la hora de evaluar una línea ascendente o descendente por años hay que tener en cuenta factores puntuales" (Rubio, 2012).

\section{Metodología}

La investigación se realizó con base en la creación de un repositorio virtual que contiene la producción científica del profesor. La página web con dirección electrónica: http:// www.educacionpersonalizante.com, perteneciente al Profesor-investigador: Martín López Calva, Investigador y escritor con doctorado en educación. 
La metodología desarrollada tomó como referente para el análisis, lo descrito en el artículo de investigación: "Análisis bibliométrico sobre la producción científica archivística en la Red de Revistas Científicas de América Latina y el Caribe (Redalyc) durante el período 2001-2011”, de Leomar José Montilla Peña. Es una investigación de tipo descriptivo que consistió en la búsqueda, registro y análisis de los documentos que dan cuenta de la producción científica del investigador, mediante una descripción de un análisis bibliométrico comprendido del año 1991 a 2017; y abordado desde la metodología cuantitativa. Se trabajó con una muestra de 103 documentos divididos en 51 artículos, 23 capítulos de libros y 29 libros en los que el investigador funge como autor, coautor o coordinador; y que pertenecen a las ramas de Educación humanista, Educación y valores, Ética profesional, pensamiento crítico y creativo e innovación educativa.

Los documentos se encontraron en diferentes bases de datos como Google Académico, Scientific Electronic Library Online (Scielo), Red de Revistas Científicas de América Latina y el Caribe, España y Portugal (Redalyc) y Educación personalizante; y revistas electrónicas como Sinéctica, Magistralis, Perfiles Educativos, Revista electrónica Didac, Revista Latinoamericana de Estudios Educativos, Extensiones, Espiral, Revista Iberoamericana sobre Calidad, Eficacia y Cambio en Educación (REICE); Mirada, Revista Electrónica de Investigación Educativa (REDIE), Ciencias Sociales y Humanidades; Entre textos, Educación y ciencia; Diálogos sobre educación,
Com Ciencia, Praxis sociología, Revista Educar y Revista Edetania. Cabe aclarar que ciertos artículos, capítulos de libros y libros solo están disponibles de manera impresa y estos no se seleccionaron para la investigación, ya que no se encuentran disponibles en las bases de datos ni revistas anteriormente mencionadas.

Para el registro de la información se elaboró una base de datos en Microsoft Excel (2013) con las siguientes categorías:

- Nombre

- Año de publicación

- Revista en la que se publicó

- País de la revista

- Resumen del artículo

- Temática basado en palabras clave y/o resumen

- Instituto del investigador

- Autores

- Tipo de revista

- Nombre del libro

- Editorial

- Nombre del capítulo

- Año de publicación

- Sinopsis

Para realizar un análisis efectivo los datos se procesaron con la herramienta computacional Microsoft Excel (2013) de la que se obtuvieron tres tablas, siete gráficas y tres figuras. 


\section{Resultados}

El estudio bibliométrico sobre artículos está fundamentado con base en la productividad autoral y coautoral; productividad de artícu- los por revistas, productividad por año, productividad de artículos por país y categorías temáticas más frecuentes.

Tabla 1. Productividad autoral y coautoral en artículos

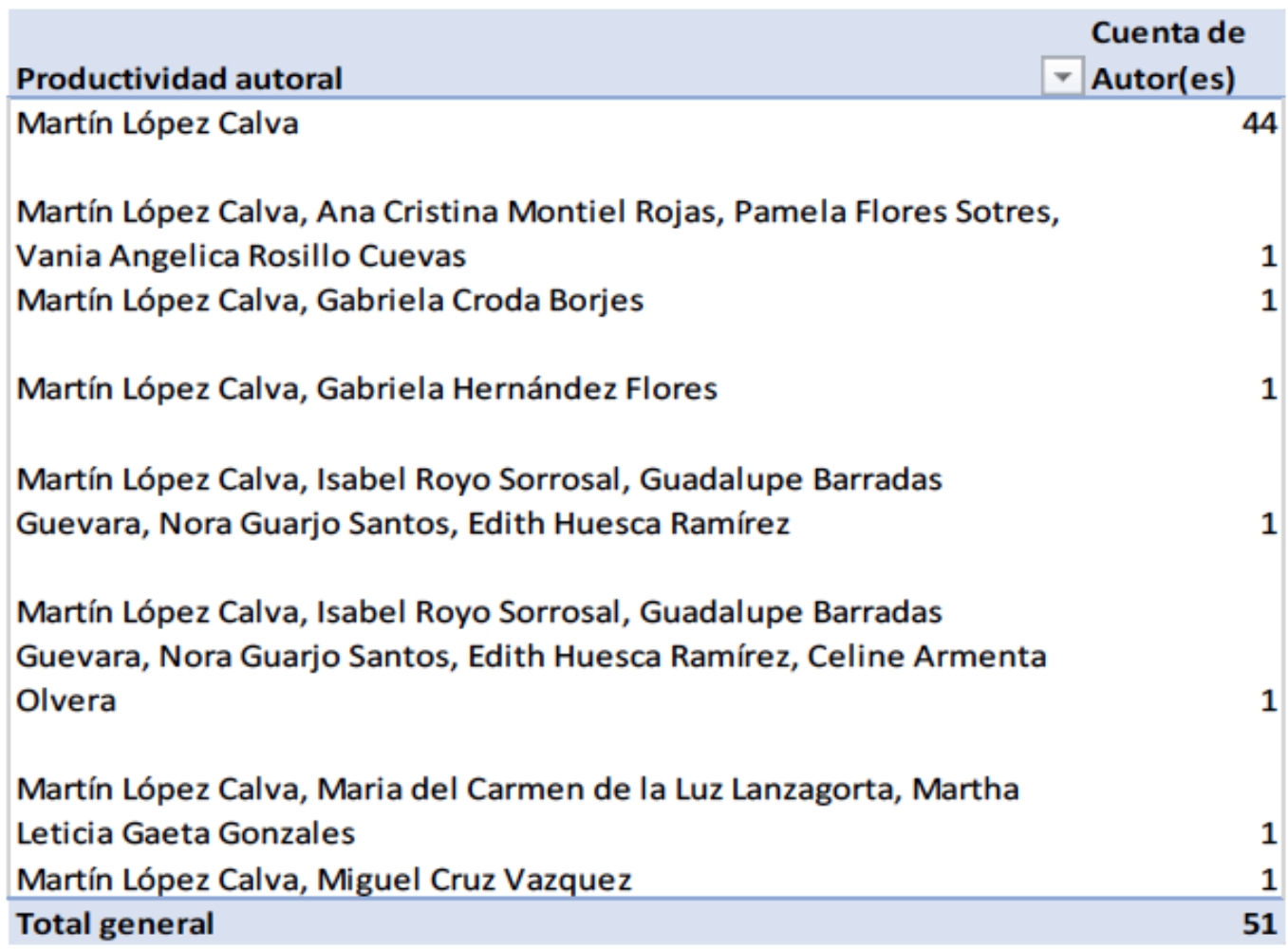

En los resultados de la productividad autoral y coautoral (ver Tabla 1), el profesor-investigador ha realizado un total 51 publicaciones, de las cuales, en 44 él ha sido autor y en 7 ar- tículos ha compartido la autoría; en 3 artículos aparecen dos autores (él y alguien más), mientras que en los 4 restantes, el investigador comparte la autoría con más de un autor. 


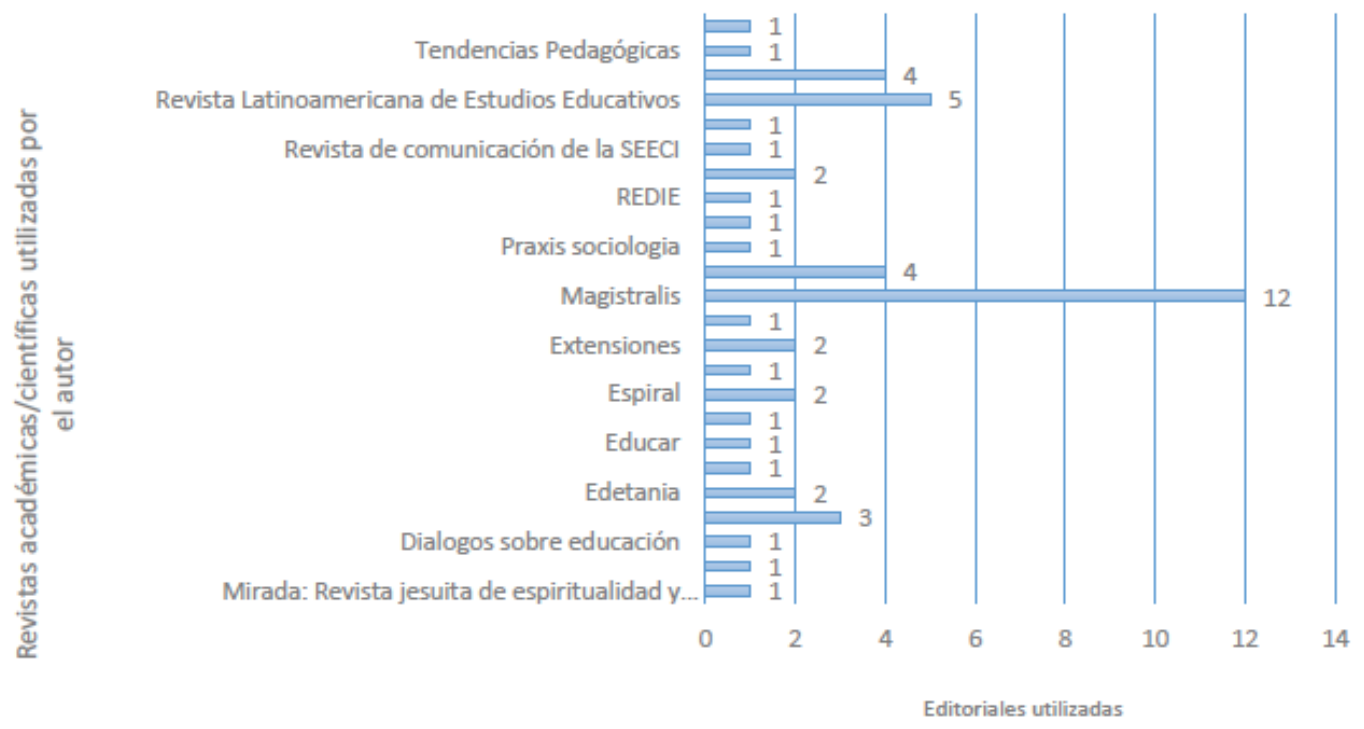

Figura 1. Productividad de artículos por Revistas

En las revistas de mayor uso, los datos analizados (ver figura 1) muestran que se utilizaron 24 revistas científicas diferentes, siendo la de mayor concurrencia la revista Magistralis (12 artículos), seguido de la Revista Latinoamericana de Estudios Educativos y la Revista Perfiles Educativos(4 publicaciones en cada una de ellas); la revista Didac, Edetania, Espiral, Extensiones y Revista Iberoamericana sobre Calidad, Eficacia y Cambio en Educación (REICE) con dos publicaciones cada una de ellas, mientras que 15 revistas se mantienen con solo una publicación.

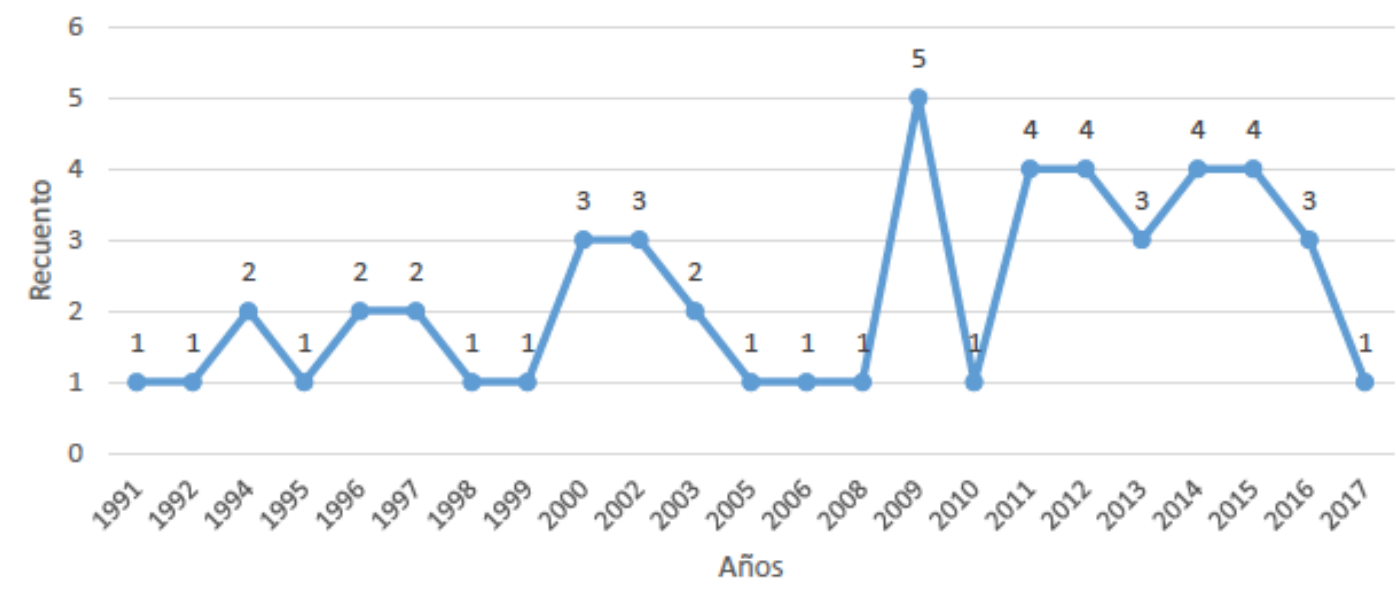

Figura 2. Productividad de artículos por año 
La producción científica por año muestra 26 años de producción científica (figura 2). El análisis indica que el año más productivo del investigador fue el 2009 con un total de cinco publicaciones. Los años que comprenden de 1991 a 2004 varía entre una y dos publicaciones, luego una declinación a una publicación por año del periodo 2005-2008. Igualmente, de cinco y cuatro publicaciones por año del 2011 al 2015; y en lo que va del 2017, solamente una publicación.
La productividad de artículos por país (ver figura 3) se basa en el país al que pertenece la revista. En esta gráfica se observa que el investigador ha publicado en revistas de cinco países diferentes, siendo México donde se concentra el mayor número de publicaciones (38); seguidos de España (8), Brasil y Colombia con dos cada uno, y Reino Unido con una publicación.

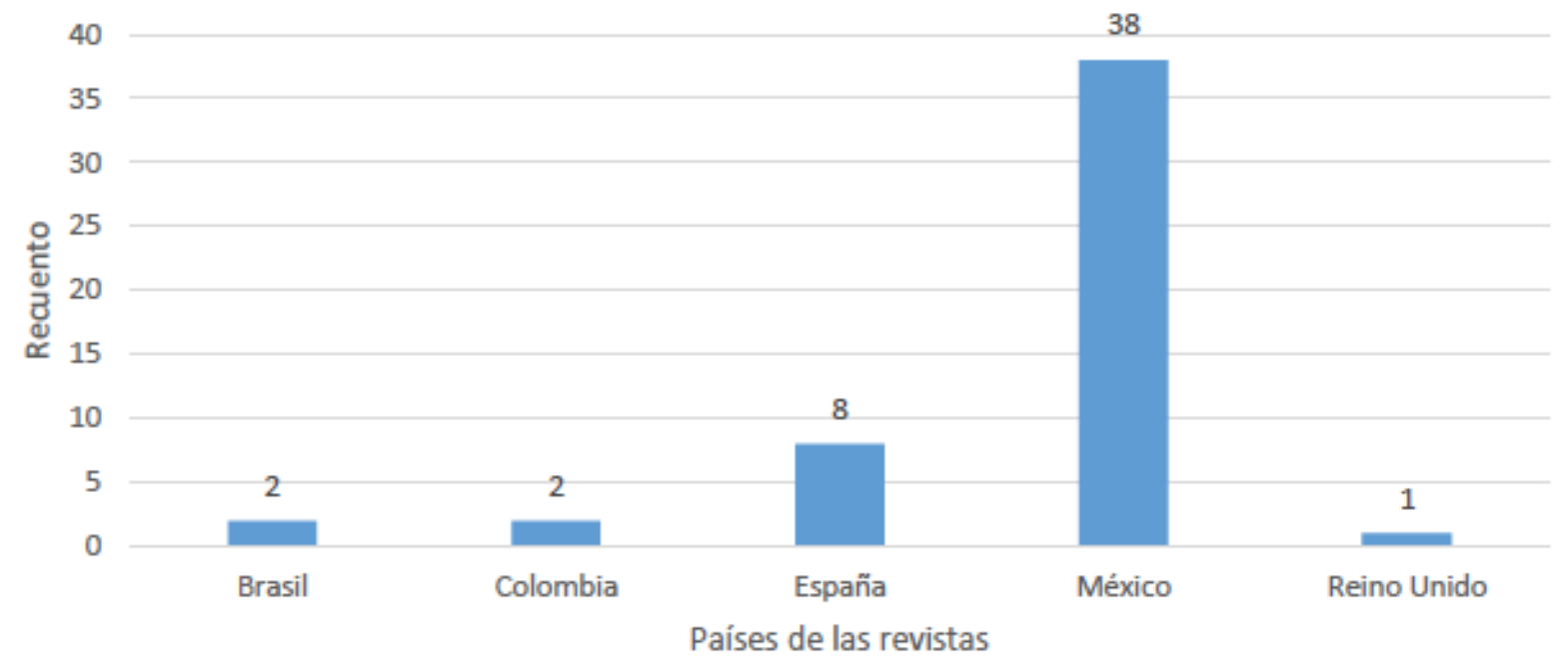

Figura 3. Productividad de artículos por país.

De los 52 artículos que se analizaron se obtuvo un total de 169 palabras clave, de las cuales solo se tomaron en cuenta las veinte con mayor índice de repetición. De las palabras utilizadas destaca en primer lugar la palabra Educación con un 30\%, siendo esta la de mayor porcentaje de uso; a continuación las palabras Ética (9\%), Ética profesional (8\%) y Valores (7\%), posteriormente, Innovación educativa
(5\%), y Filosofía de la Educación; Complejidad, formación profesional y Humanismo con $3 \%$ cada una.

El estudio bibliométrico sobre capítulos de libros se fundamenta con base en la productividad autoral y coautoral; productividad por editorial, productividad por año y temáticas más frecuentes. (Ver figura 4). 


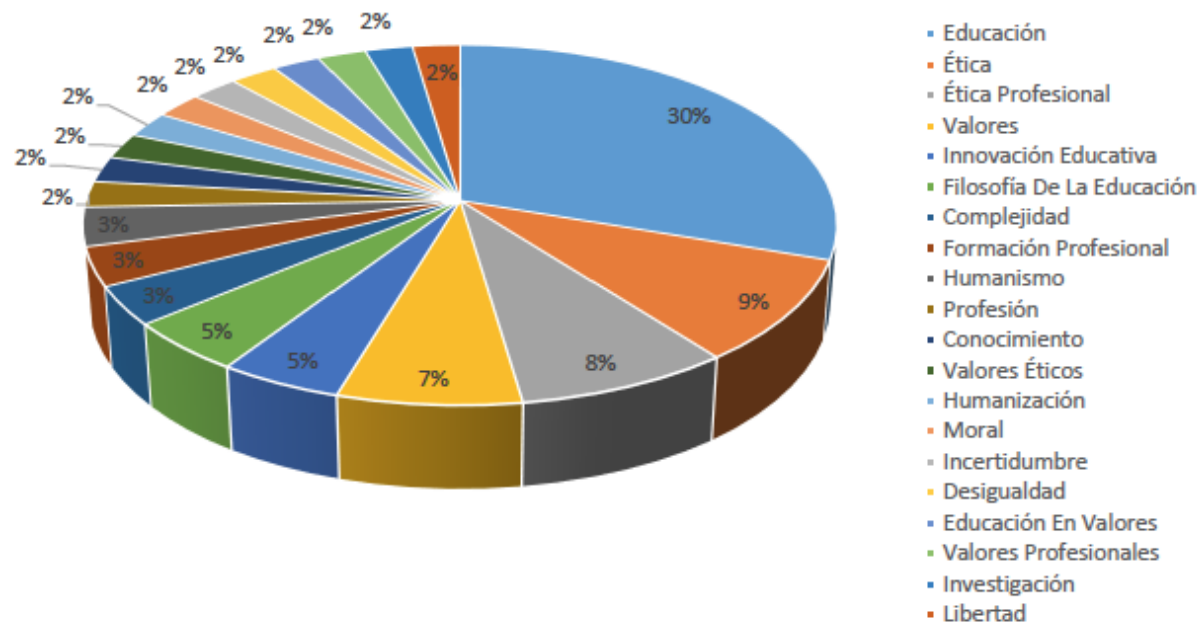

Figura 4. Temáticas más frecuentes en artículos

Tabla 2. Productividad autoral y coautoral en capítulos de libros

\begin{tabular}{|lr|}
\hline Productividad autoral & Cuenta de \\
\hline Juan Martín López Calva, Luz Del Carmen Montes Pacheco, En & 1 \\
Martín López Calva & 14 \\
Martin Lopez Calva, Maria Isabel Royo Sorrosal, Guadalupe B & 1 \\
Martín López Calva, Gabriela Croda Borges & 1 \\
Martín López Calva, Isabel Royo Sorrosal, Guadalupe Barrada: & 1 \\
Martín López Calva, Laura Bárcenas Pozos, Eneida Nora Guaja & 1 \\
Martín López Calva, Marisol Aguilar Mier & 1 \\
Martín López Calva, Martha Leticia Gaeta González, Judith Ág| & 1 \\
Martín López Calva, Martha Leticia Gaeta González, María del & 1 \\
Martín López Calva, Paulina Iturbide Fernandez, Victoria Carc & 1 \\
(en blanco) & 23 \\
\hline Total general &
\end{tabular}

El investigador cuenta con un total de 23 capítulos de libros, de los cuales catorce pertenecen a su autoría. En dos capítulos el investigador comparte la autoría con una persona; posteriormente, en dos trabajos el investiga- dor comparte la autoría con dos personas, siendo un total de tres autores; mientras que en los cuatro restantes, el investigador comparte con más de tres personas. 


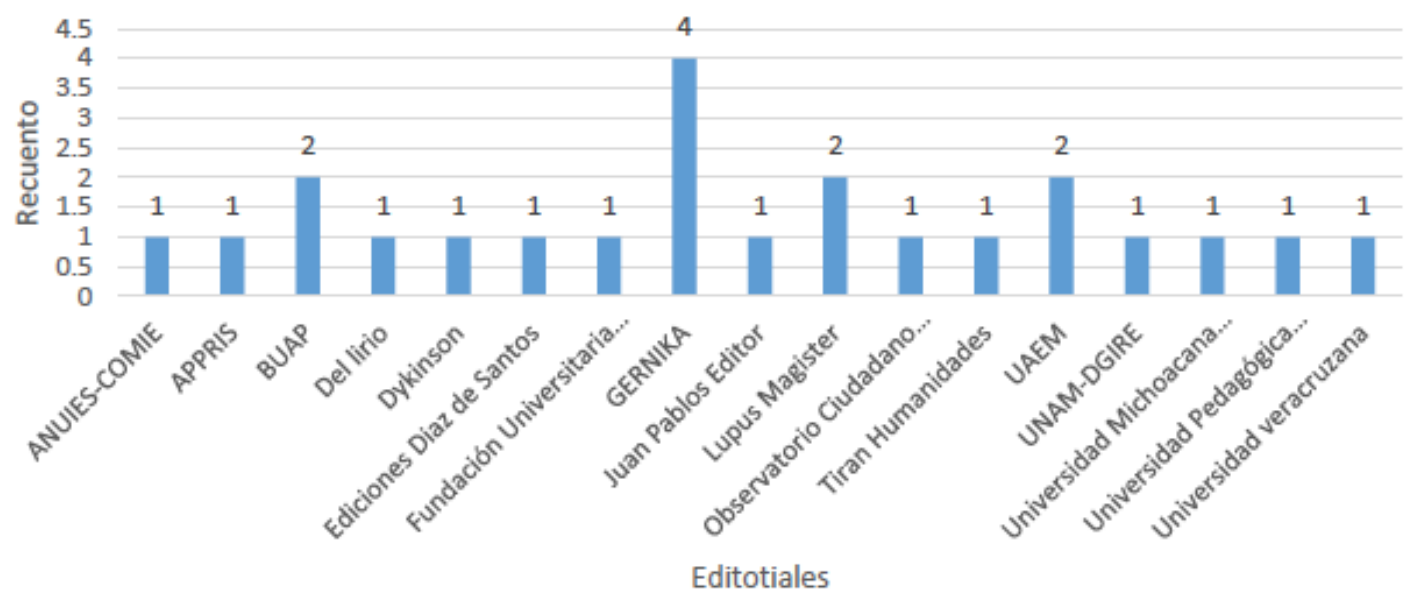

Figura 5. Productividad de capítulos de libros por editorial

La productividad por editoriales, el investigador ha utilizado 17 editoriales diferentes, siendo la editorial GERNIKA la de mayor frecuencia con cuatro publicaciones, seguidas de la Universidad Autónoma del Estado de Morelos (UAEM), Lupus Magister y Benemérita Universidad Autónoma de Puebla (BUAP) en México con dos apariciones en cada una. Las trece editoriales restantes cuentan con solo una publicación de las que cabe mencionar dos co-ediciones con la Asociación Nacional de Universidades e Instituciones de Educación Superior (ANUIES) con el Consejo Mexicano de Investigación Educativa A.C (COMIE); y Universidad Nacional Autónoma de México (UNAM) con la Dirección General de Incorporación y Revalidación de Estudios (DGIRE).

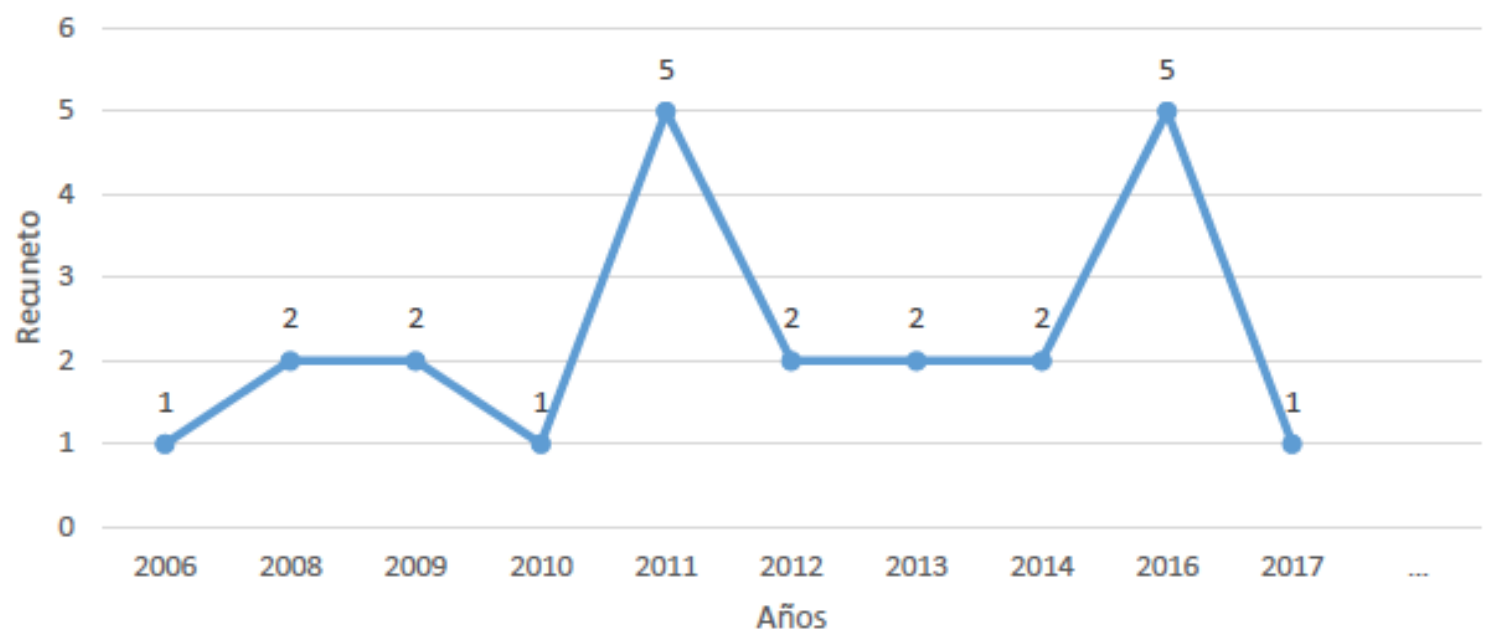

Figura 6. Productividad de capítulos de libros por año.

En la productividad por año, se observa dos máximos de cinco publicaciones de capítulos de libros en los años 2011 y 2016. Cabe resaltar que su primera aparición en este tipo de publicaciones en el año 2006, después tiene dos contribuciones (2008 y 2009); y pos- teriormente, una sola publicación en el año 2010; después descendió a dos publicaciones entre los años 2012 y 2014; de nuevo alcanza su máximo en el año 2015, y disminuye a solo una contribución en lo que va del año 2017. 


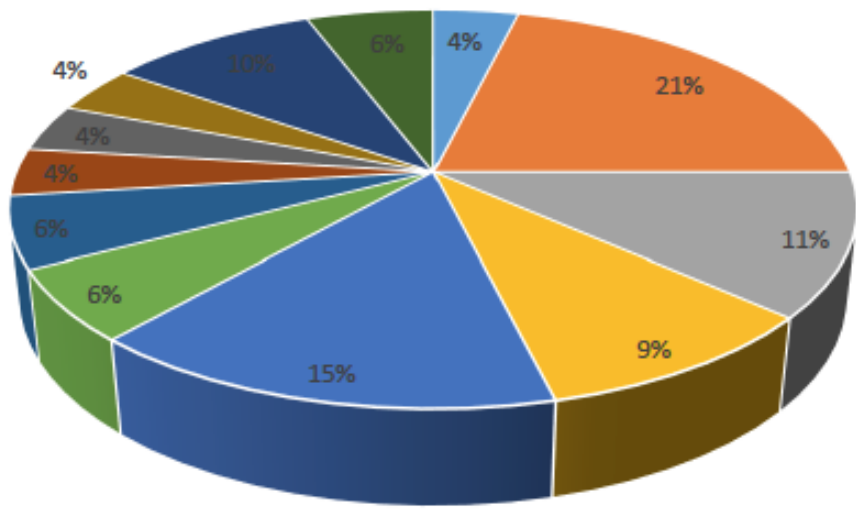

- Competencias éticas

- Educación

- Ética

" Ética planetaria

- Ética profesional

- Formación ciudadana

- Formación profesional

- Humanización

- Moral

- Responsabilidad social

- Valores

- Valores profesionales

Figura 7. Productividad de capítulos de libros por año.

En cuanto a palabras claves utilizadas con mayor frecuencia en los capítulos de libros se contabilizaron y catalogaron 99, de las cuales solo se tomaron en cuenta las diez con mayor índice de repetición. La de mayor frecuencia es la palabra Educación (21\%), seguida de Ética profesional (15\%), Ética (12\%), Ética planetaria (9\%); formación profesional y valores profesionales con $6 \%$ cada una de ellas.
El análisis bibliométrico sobre libros se encuentra fundamentado con base en la productividad autoral y coautoral; productividad por editorial, productividad por año y temáticas más frecuentes.

Tabla 3. Productividad autoral y coautoral en libros

\begin{tabular}{|l|c|}
\hline Productividad autoral & $\begin{array}{c}\text { Cuenta de } \\
\text { Autor (es) }\end{array}$ \\
\hline Martín López Calva, Lorena Barquín García-Villoslada & 2 \\
\hline Martín López Calva, Rafael de Regil Vélez & 1 \\
\hline Martín López Calva & 19 \\
\hline Martín López Calva, Ana Esther Escalante Ferrer, Arturo Benítez Zavala & 1 \\
\hline $\begin{array}{l}\text { Martín López Calva, Enrique Luengo González, Laura Orellana Trinidad, Javier Sánchez } \\
\text { Díaz de Rivera, Sylvia Schmelkes del Valle, Fernando Fernandez Font, Alberto Alvarez } \\
\text { Gutiérrez, Alfredo Castillo Romero, Sergio Cobo González }\end{array}$ & 1 \\
\hline Martin Lopez Calva, Judith Cavazos Arroyo, Martha Leticia Gaeta Gonzalez & 1 \\
\hline Martín López Calva, Lorena Barquín García-Villoslada & 2 \\
\hline Martín López Calva, Martha Leticia Gaeta Gonzalez & 1 \\
\hline $\begin{array}{l}\text { Martín López Calva, Nelly Milady López Rodríguez, Alberto Aguilar Alvarez, Juan An- } \\
\text { tonio García Fraile }\end{array}$ & 1 \\
\hline Total general & $\mathbf{2 9}$ \\
\hline
\end{tabular}


El investigador cuenta con 29 libros publicados, de los cuales diecinueve aparece como único autor. Se observa que publicó seis libros donde el profesor comparte la autoría con una persona; en el resto de publicaciones, el investigador comparte la autoría con más de dos autores.

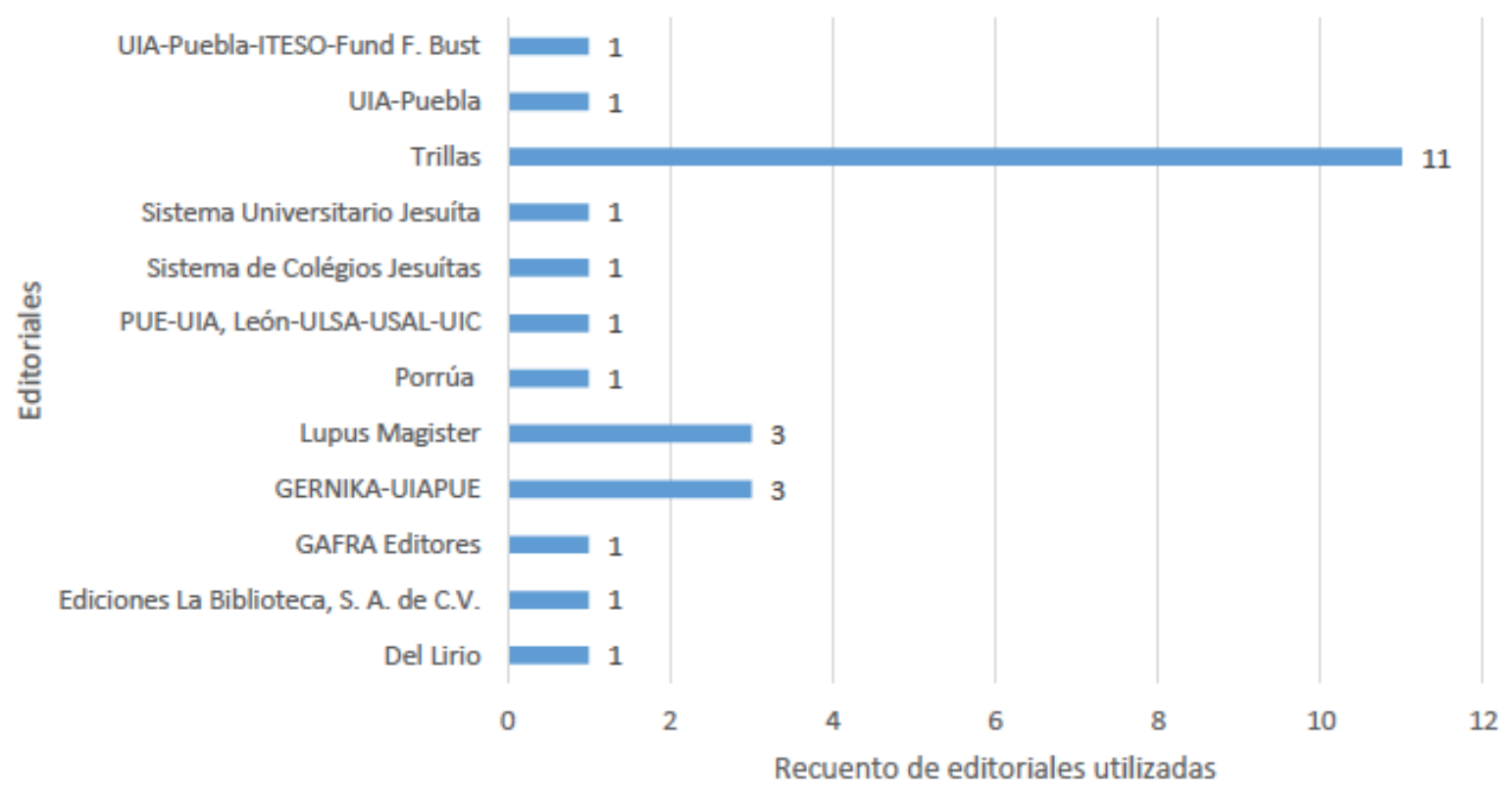

Figura 8. Productividad de libros por editorial.

Las editoriales utilizadas suman catorce, la de mayor frecuencia corresponde a Trillas con once publicaciones, seguidas de GERNIKA (5) de las cuales, tres con co-edición de la Universidad Iberoamericana Puebla (UIA-
PUE). Lupus Magister cuenta con tres publicaciones, Ediciones la Biblioteca S.A. de C.V. cuenta con dos; mientras que nueve editoriales solo cuentan con una publicación.

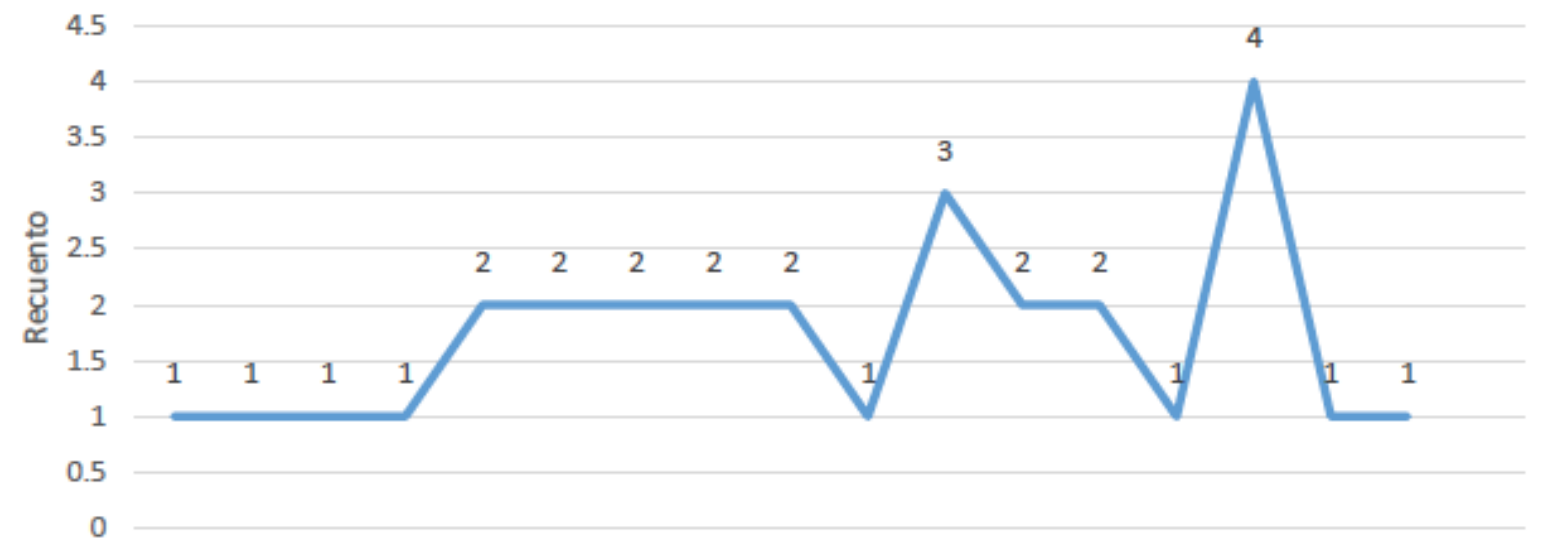

19941997199920002001200320052006200720082009201020112012201420152017

Año

Figura 9. Productividad de libros por año en libros. 
En cuanto a la productividad de libros por año, el profesor-investigador comenzó en el año 1994, teniendo un crecimiento gradual de un libro publicado al año siguiente (1995); dos libros por año desde el año 2000 al 2004; mientras que en el año 2007 con cuatro libros. Bajó la productividad en el 2008 con un libro, pero en el 2014 alcanzó su máximo con cuatro libros publicados para después descender a una publicación en el año 2016. En los años 2006 y 2014, el investigador ha variado en sus números de publicaciones.

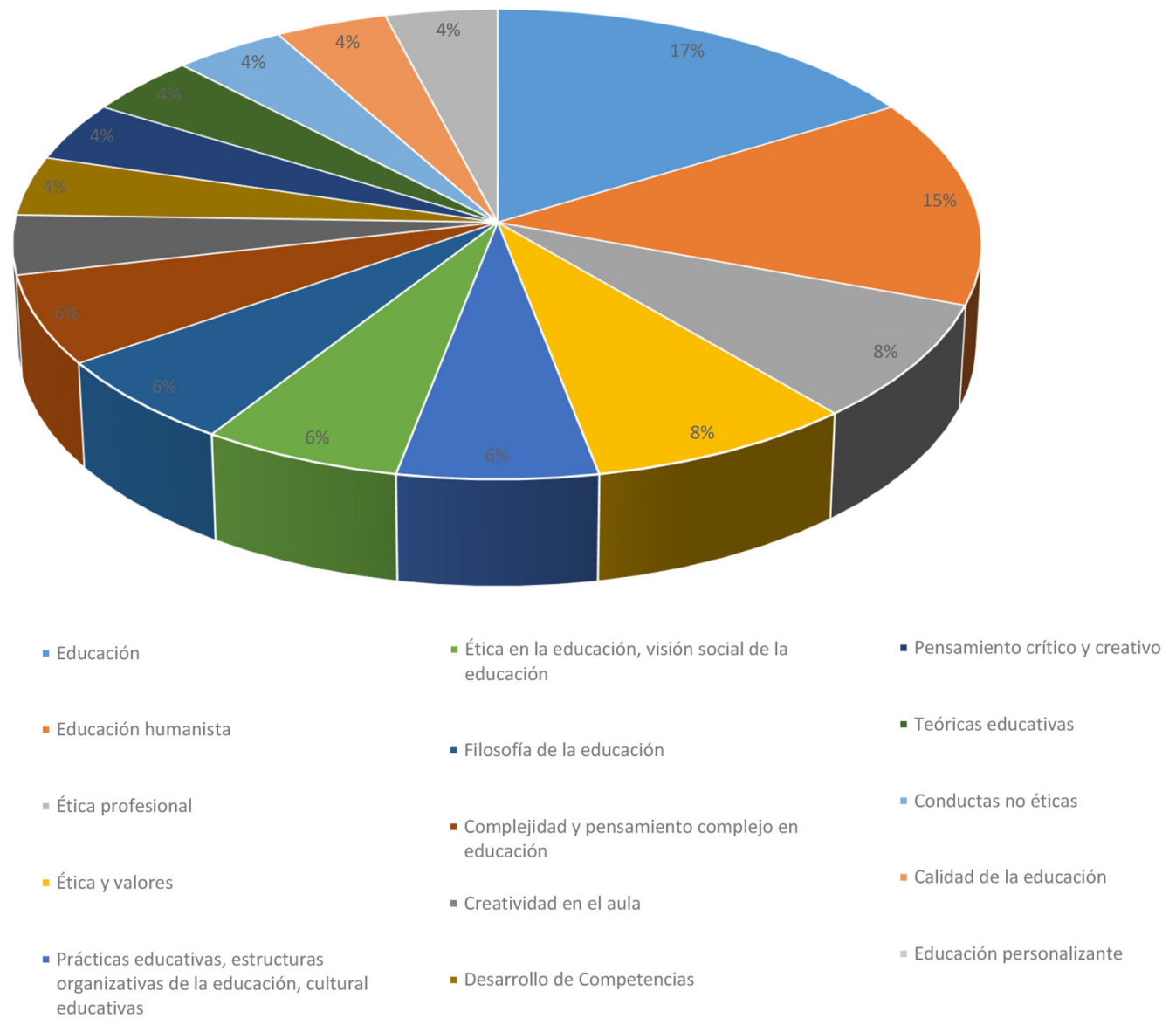

Figura 10. Temáticas más frecuentes en los libros

A partir de la revisión de la sinopsis de los libros publicados se extrajeron palabras clave que, posteriormente, se clasificaron teniendo un total de 94 palabras, de las cuales solo se seleccionaron las quince con mayor índice de repetición. Cabe mencionar que se utilizó un vocabulario controlado y mesurado para mantener la objetividad. Del total las palabras que se utilizan con mayor frecuencia es Educación (17\%), Educación humanista (15\%), Ética y valores y Ética profesional (8\% cada una). Prácticas educativas, visión social de la educación, Ética en la educación, Filosofía de la educación y complejidad y pensamiento complejo con $6 \%$. 


\section{Discusión}

El análisis bibliométrico sobre la producción científica del investigador educativo en el periodo de tiempo de 1991-2017, pone en evidencia la prolífica producción científica y académica del investigador en cuestión durante veintiséis años. Constituye, también, una aportación significativa al conocimiento científico en general.

De los 103 trabajos analizados, el profesor aparece en 77 de sus publicaciones totales como único autor, lo que revela una tendencia a la poca colaboración coautoral. De acuerdo a los datos obtenidos el investigador trata los temas de Educación humanista, Educación y valores, Ética profesional, pensamiento crítico y creativo e innovación educativa, ya que cada esas palabras aparecen con frecuencia tanto en sus artículos publicados como en sus capítulos de libros y en libros. De esta forma se puede observar que el profesor tiene una tendencia definida a la investigación de la Educación, la Ética y los Valores.

Un dato interesante es el aumento de la producción científica de forma gradual a través de los años; este fenómeno se observa cuando se trata de análisis bibliométricos de revista, países e instituciones. Sin embargo, se pudo observar que la producción científica del investigador aumentaba gradualmente conforme pasaban los años desde su primera publicación hasta decaer en los años recientes. No obstante, en los periodos de poca publicación, hay que tener en cuenta factores puntuales tales como sociales, políticas, so- cioeconómicas, culturales que pueden afectar el nivel de producción científica del profesorinvestigador. El aumento o disminución del nivel de contribuciones puede ser objeto de una investigación futura.

La productividad de artículos científicos en revistas mexicanas prevalece, teniendo como segundo lugar a las revistas españolas. En cuestión de las revistas y las editoriales, el investigador publica con mayor regularidad en la revista Magistralis, en el caso de las editoriales GERNIKA y Trillas figuran como las más frecuentes.

El análisis bibliométrico realizado muestra información que puede ser de mucha utilidad para el investigador educativo, ya que puede observar sus líneas de investigación y tomar decisiones para profundizar en temas que han sido poco abordados. De igual modo tienen un gran valor para otros académicos y estudiantes dentro de la literatura científica, ya que dicho análisis puede orientar al lector que sigue la producción del profesor a hacer un seguimiento sistemático de su larga trayectoria, a visualizar las temáticas de mayor acogida, el índice de colaboración, las editoriales más concurrentes y, en última instancia, despertar el interés para adentrarse a las líneas de investigación que maneja el investigador académico. 


\section{Referencias}

Arroyo, A. A., Pulgarín, A. y Gil-Leiva, I. (2006). Análisis bibliométrico de la producción científica de la Universidad Politécnica de Valencia 1973-2001. Revista española de Documentación Científica, 29 (3), p. 345

Agudelo, D., Bretón López, J. y Buela Casal, G. (2003). Análisis bibliométrico de las revistas de Psicología Clínica editadas en castellano. Psicothema, pp. 507-516.

Díaz Mujica, D. (2007). Análisis bibliométrico de la revista Archivos Latinoamericanos de Nutrición. In Anales venezolanos de Nutrición, pp. 22-29.

Documentación, C. d. (1986-1987). La producción científica de la universidad española en ciencias sociales y humanidades. Madrid: Instituto de Información y Documentación en Ciencias Sociales y Humanidades, Madrid.

Escorcia-Otálora, T. A. (2008). Análisis bibliométrico de los artículos originales publicados en. Pontificia Universidad Javeriana, UNIVERSITAS SCIENTIARUM, pp. 236-244.

Lotka, A. J. (1926). The frecuency Distribution of Scientific Productivity. Journal of the Washington Academy of Sciences, p. 317.

Montilla, L. J. (2012). Análisis bibliométrico sobre la producción científica archivística en la Red de Revistas Científicas de América Latina y el Caribe (Redalyc) durante el período 2001-2011. Biblios, p. 48.

Price, D. S. (1963). Little Science, Big Science. New York: Columbia University Press.

Pritchard, A. (1969). Statiscal bibliography or Bibliometrics. Journal of Documentation, pp. 348-369.

Rubio, M. (8 de Junio de 2012). Bibliometría y ciencias sociales. Recuperado de http://clio.rediris.es/clionet/articulos/bibliometria.htm

Rueda-Clausen, C., Villa-Roel, C. y Rueda-Clausen, C. (2005). Indicadores bibliométricos: origen, aplicación, contradicción. MedUNAB, pp. 29-36. 